\title{
Blockchain and cryptocurrencies: economic and financial research
}

\author{
Alessandra Cretarola ${ }^{1} \cdot$ Gianna Figà-Talamanca $^{2} \cdot$ Cyril Grunspan $^{3}$ \\ Accepted: 25 October 2021 / Published online: 13 November 2021 \\ () The Author(s), under exclusive licence to Associazione per la Matematica Applicata alle Scienze Economiche \\ e Sociali (AMASES) 2021
}

\begin{abstract}
The motivation of proposing and editing the Special Issue "Blockchain and cryptocurrencies" came from the inspirational invited and contributed talks at the 43rd annual A.M.A.S.E.S. conference held in Perugia in September 2019. All the papers have gone through the journal regular refereeing process under the same standards set by the journal, and nine contributions were finally accepted for publication.
\end{abstract}

\section{Literature background}

Bitcoin, the pioneer cryptocurrency, was conceived in 2008 by an individual or a group of researchers under the pseudonymous of Nakamoto (2008) and implemented in 2009 as an electronic payment system via a breakthrough application of the blockchain technology; it is based on an open-source software which generates a peer-to-peer network and does not rely on central banks to regulate the money supply, and it enables essentially anonymous transactions. Two major technological advances set Bitcoin apart in the world of cryptocurrencies:

- the extensive and repeated use of proof of work;

- the invention of smart contracts.

$凶$ Gianna Figà-Talamanca

gianna.figatalamanca@unipg.it

Alessandra Cretarola

alessandra.cretarola@unipg.it

Cyril Grunspan

cyril.grunspan@devinci.fr

1 Department of Mathematics and Computer Science, University of Perugia, via L. Vanvitelli 1, 06123 Perugia, Italy

2 Department of Economics, University of Perugia, via A. Pascoli 20, 06123 Perugia, Italy

3 Léonard de Vinci Pôle Universitaire, Research Center, 92916 Paris, La Défense Cedex, France 
The first advance (proof of work) establishes a link between computer security and probability theory (specifically, Poisson processes). Prior to Satoshi Nakamoto's work, there have been attempts at creating decentralized cryptocurrency networks with secure transactions. Nakamoto's model represents a different paradigm, where double-spending attacks — spending the same amount twice — are unlikely, provided some requirements are met (Grunspan and Pérez-Marco 2018). Such repeated attacks prove to be unprofitable, unless one is ready to double-spend enormous amounts on a regular basis - an unreasonable assumption in practice (Grunspan 2021). This is achieved using the proof of work concept, which originates in the design of spam filters (Dwork and Naor 1993).

The second advance (strangely absent from Bitcoin's founding paper, which only focuses on describing the protocol itself) turns Bitcoin into a truly programmable currency. It allows for the creation of payment channels between users which, combined, give rise to the Lightning Network, an overlay to the Bitcoin network (Dryja and Poon 2015). This new network has incredible performance in terms of scalability, and its transaction throughput (number of transactions processed per second) is far superior to that of traditional centralized networks like Visa or Paypal.

Smart contracts also pave the way to a truly decentralized finance (DeFi). One example is Ethereum, another cryptocurrency created in 2015 in the wake of Bitcoin thanks to Solidity (its flexible programming language). This new finance handles volumes of about 100 billion today, and is growing rapidly ( $+400 \%$ in one year). DeFi relies on new offerings that were never implemented in traditional markets. For instance, Automated Market Makers are truly decentralized trading platforms (Angeris and Chitra 2020). Defi also offers new products such as flash loans or staking derivatives for cryptocurrencies like Solana or Ethereum 2.0 based on a protocol using PoS (Chitra and Evans 2020). The link with traditional finance is made through "stable coins"-crypto-currencies whose price is pegged to the US dollar.

The academic response to this economic and financial breakpoint was initially hesitant and cautious but eventually raised in 2016, and was especially boosted by the exponential price increase in 2017. First economic studies focused on framing Bitcoin among fiat currencies, commodities, or stocks, trying to identify its intrinsic value, see Nian and Chuen (2015), Yermack (2015), Cheah and Fry (2015), Bjerg (2016) and Böhme et al. (2015). Then, the advent of thousands of new cryptocurrencies has raised several new research questions: are there specific stylized facts, how are cryptocurrencies related to traditional financial assets, how can they be used to get profitable trading strategies, how are they related to one another, are there common driving factors? Indeed, Bitcoin and other cryptocurrencies are currently seen as an alternative investment class, and, though their high-returns/high-volatility profile has initially motivated speculative investments (Yermack 2015; Baur et al. 2018), more recent studies claim that investing in these assets does improve the overall portfolio diversification (Platanakis et al. 2018). One of the main strands of research in Bitcoin and cryptocurrencies is represented by the econometric modeling of the price dynamics and the identification of possible driving factors. Some contributions, see e.g., Dyhrberg (2016), describe the relationship between Bitcoin returns and factors in the traditional financial market, such as the price of gold, gold futures, and stock market indexes. Moreover, Kristoufek (2015) investigates the dependence of Bitcoin 
price on crypto-related factors, such as the hash-rate, the mining difficulty, the number of transactions and the total number of Bitcoin in circulation. Many researches suggest that Bitcoin returns and volatility are driven by investor attention, sentiment or by specific measures of market attractiveness, see e.g., Ciaian et al. (2016), Figa-Talamanca and Patacca (2019), Ahn and Kim (2019), Eom et al. (2019) and Figà-Talamanca and Patacca (2020). Notably, the occurrence of periods of hype for cryptocurrencies, followed by periods of skepticism, has encouraged the analysis of jumps, structural breaks and bubble effects in their price dynamics, see Garcia et al. (2014), Cheah and Fry (2015), Fry and Cheah (2016), Corbet et al. (2018b), Bouri et al. (2019), Cretarola and Figà-Talamanca (2019), Scaillet et al. (2020), Hafner (2020) and Cretarola and Figà-Talamanca (2020), among others. The relationship between different cryptocurrencies being very strong, most of the above contributions focus on Bitcoin as a benchmark for the whole asset class. The interdependence between cryptocurrencies is investigated in Ciaian and Rajcaniova (2018), Yaya et al. (2019), Chaim and Laurini (2019), Kumar and Anandarao (2019), Mensi et al. (2019), Blau et al. (2020), Tiwari et al. (2020) and Figà-Talamanca et al. (2021), by applying several dependence concepts and alternative methodologies. After the introduction of Bitcoin Futures in the Chicago Board Option Exchange, a few papers have also appeared on the evaluation of Bitcoin derivatives, see Chen et al. (2018), Corbet et al. (2018a), Cretarola et al. (2020) and Siu and Elliott (2021), whereas investments, trading strategies and possible arbitrage opportunities are discussed in Lintilhac and Tourin (2017), Nakano et al. (2018), Bistarelli et al. (2019), Bistarelli et al. (2019b) and Shynkevich (2021).

\section{Special issue contributions}

The collected papers span several topics, most of them through econometric analyses: the modeling of returns and volatility dynamics; the identification of profitable investment strategies; the study of the trading volume of cryptocurrencies; the post-sale behavior of tokens issued through initial coin offerings as well as the development of the overall coins and tokens industry.

Majdoub et al. (2021) attempts to investigate whether Bitcoin can be hedged by selected fiat currencies (EUR, JPY, and GBP). The authors compute optimal hedge ratios between Bitcoin and fiat currencies over the period February 2012-November 2017 based on the VAR-DCC-GARCH model, VAR-ADCC-GARCH model and VAR-component GARCH-DCC model. They evidence that the correlations between Bitcoin and fiat currencies have a time-varying dynamic under different model specifications. Thereafter, they propose suitable dynamic hedging strategies when investing in the Bitcoin market.

The relationship between volatilities of five cryptocurrencies, American indices (SP500, Nasdaq, and VIX), oil, and gold is analyzed in Ghorbel and Jeribi (2021) in a multivariate BEKK-GARCH model framework. A higher volatility spillover is detected between cryptocurrencies than between cryptocurrencies and other financial assets.

Kyriazis (2021) investigates the nexus between cryptocurrencies connected to cannabis production and the three highest capitalization digital currencies, Bitcoin, 
Ethereum and Ripple. The GARCH, EGARCH, TGARCH and GJR-GARCH specifications are employed in order to analyze volatility characteristics. Findings reveal that GARCH and GJR-GARCH specifications are most appropriate to explain the volatility of each cannabis cryptocurrency. This allows to recognize the existence of thresholds in the volatility of cannabis cryptocurrencies when examining their nexus with major digital currencies.

The multivariate relationship between cryptocurrencies is investigated in FigàTalamanca et al. (2021) by applying a dynamic factor analysis to the joint behavior of Bitcoin, Ethereum, Litecoin and Monero, as a representative basket of the cryptocurrencies asset class, from 2016 to 2019. The authors show that the basket price is suitably described by a model with two dynamic factors, of which the first is integrated and the second is stationary until the end of August 2019. Based on this evidence, they introduce a trading strategy which proves profitable only when the second factor is stationary.

Angelis et al. (2021) propose innovative profit-oriented trading strategies on Bitcoins for risk-seeking investors, which are based on buying or selling the so-called Contracts for Difference. Moreover, thanks to the use of a proper machine/deep learning model for predicting aims, i.e., a recurrent neural network with a long short-term memory, the authors are able to forecast possible investment scenarios under a suitable theoretical model specification.

The problem of forecasting the intraday short-term volume and its uncertainty in exchange markets for cryptocurrencies is studied in Antulov-Fantulin et al. (2021). Precisely, the predictions are built by using transaction and order book data from different markets, and a temporal mixture ensemble model is proposed to identify, at each time step, the set of data which is locally most useful for the forecasting. The authors obtain some empirical findings on the outperformance of their model with respect to time series models both in point and in interval predictions of trading volume. In addition, they also evidence similar results when conditioning to the volume quartile.

In Provenzano and Baggio (2021), a wide application of complex network analysis to cryptocurrencies markets is provided. Specifically, the authors characterize the dynamics and estimate the synchronicity between the price and volume series of three cryptocurrencies, Bitcoin, Ethereum, and Litecoin. The results show similar complex structures in terms of number and internal composition of communities. In particular, cyclical patterns of similar wavelength and amplitude, and a different degree of synchronization before and after a collapse event over the period considered can be observed in the price and volume dynamics of the three cryptocurrencies, suggesting possible investment opportunities.

The novel funding mechanism of initial coin offerings (ICOs), where digital tokens are issued and sold to investors, is the subject of research of Ante and Meyer (2021). In particular, they investigate the price effects of 250 cross-listing events of 135 individual tokens and possible abnormal returns when they are immediately traded on secondary markets. They find significant abnormal returns of $6.51 \%$ on the listing day and $9.97 \%$ over a seven-day window around the event; these abnormal returns are also affected by the exchanges on which the listings occur and by liquidity-related metrics. 
Finally, Gandal et al. (2021) analyze the flourishing industry of cryptocurrency coins and tokens. Even though these terms are commonly used as interchangeable, they are very different in nature and deserve a separate analysis. While cryptocurrency coins have the purpose to replace fiat currency as a store of value, the main use of a token is to fund a blockchain-based project through an ICO. The authors investigate the creation, competition, and destruction of 1082 coins and 725 tokens during the period 20132018 and find that $44 \%$ of publicly traded coins are abandoned, at least temporarily; $71 \%$ of abandoned coins are later resurrected; $18 \%$ of coins fail permanently. An unexpected finding is that the bursting of the 2017 Bitcoin bubble has not affected the rise of alternative cryptocurrencies.

\section{References}

Ahn, Y., Kim, D.: Sentiment disagreement and bitcoin price fluctuations: a psycholinguistic approach. Appl. Econ. Lett., pp. 1-5 (2019)

Angeris, G., Chitra, T.: Improved price oracles: constant function market makers. In: Proceedings of the 2nd ACM Conference on Advances in Financial Technologies, Association for Computing Machinery, pp. 80-91 (2020)

Ante, L., Meyer, A.: Cross-listings of blockchain-based tokens issued through initial coin offerings: Do liquidity and specific cryptocurrency exchanges matter? Decis. Econ. Finance, pp. 1-24 (2021)

Antulov-Fantulin, N., Guo, T., Lillo, F.: Temporal mixture ensemble models for probabilistic forecasting of intraday cryptocurrency volume. Decis. Econ. Finance, pp. 1-36 (2021)

Baur, D.G., Hong, K., Lee, A.D.: Bitcoin: Medium of exchange or speculative assets? J. Int. Finan. Markets. Inst. Money 54, 177-189 (2018)

Bistarelli, S., Cretarola, A., Figà-Talamanca, G., Mercanti, I., Patacca, M.: Is arbitrage possible in the bitcoin market? (Work-in-progress paper). In: Coppola M, Carlini E, D’Agostino D, Altmann J, Bañares JÀ (eds) Economics of Grids, Clouds, Systems, and Services. GECON 2018. Lecture Notes in Computer Science, vol. 11113., Springer, Cham, pp. 243-251 (2019a)

Bistarelli, S., Cretarola, A., Figà-Talamanca, G., Patacca, M.: Model-based arbitrage in multi-exchange models for bitcoin price dynamics. Digit. Finance 1(1), 23-46 (2019b)

Bjerg, O.: How is bitcoin money? Theory, Culture \& Society 33(1), 53-72 (2016)

Blau, B., Griffith, T., Whitby, R., et al.: Comovement in the cryptocurrency market. Economics Bulletin 40(1), 448-455 (2020)

Böhme, R., Christin, N., Edelman, B., Moore, T.: Bitcoin: Economics, technology, and governance. J. Econ. Perspect. 29(2), 213-238 (2015)

Bouri, E., Gil-Alana, L.A., Gupta, R., Roubaud, D.: Modelling long memory volatility in the bitcoin market: evidence of persistence and structural breaks. Int. J. Finance Econ. 24, 412-426 (2019)

Chaim, P., Laurini, M.P.: Nonlinear dependence in cryptocurrency markets. N. Am. J. Econ. Finance 48, 32-47 (2019)

Cheah, E.T., Fry, J.: Speculative bubbles in bitcoin markets? an empirical investigation into the fundamental value of bitcoin. Econ. Lett. 130, 32-36 (2015)

Chen, C. Y. H., Härdle, W. K., Hou, A. J., Wang, W.: Pricing cryptocurrency options: the case of CRIX and Bitcoin. Tech. rep., IRTG 1792 Discussion Paper (2018)

Chitra, T., Evans, A.: Why stake when you can borrow? (2020). arXiv:2006.11156

Ciaian, P., Rajcaniova, M., Kancs, D.: The economics of bitcoin price formation. Appl. Econ. 48(19), 1799-1815 (2016)

Ciaian, P., Rajcaniova, M., et al.: Virtual relationships: Short-and long-run evidence from Bitcoin and altcoin markets. J. Int. Finan. Markets. Inst. Money 52, 173-195 (2018)

Corbet, S., Lucey, B., Peat, M., Vigne, S.: Bitcoin futures-what use are they? Econ. Lett. 172, 23-27 (2018a)

Corbet, S., Lucey, B., Yarovaya, L.: Datestamping the bitcoin and Ethereum bubbles. Financ. Res. Lett. 26, 81-88 (2018b)

Cretarola, A., Figà-Talamanca, G.: Detecting bubbles in bitcoin price dynamics via market exuberance. Ann. Oper. Res. 299, 1-21 (2019) 
Cretarola, A., Figà-Talamanca, G.: Bubble regime identification in an attention-based model for bitcoin and Ethereum price dynamics. Econ. Lett. 191, 108831 (2020)

Cretarola, A., Figà-Talamanca, G., Patacca, M.: Market attention and bitcoin price modeling: theory, estimation and option pricing. Decis. Econ. Finan. 43(1), 187-228 (2020)

De Angelis, P., De Marchis, R., Marino, M., Martire, A. L., Oliva, I.: Betting on bitcoin: a profitable trading between directional and shielding strategies. Decis. Econ. Finance pp 1-21 (2021)

Dryja, T., Poon, J.: Lightning network (2015). https://lightning.network/

Dwork, C., Naor, M.: Pricing via processing or combatting junk mail. In: Brickell, E.F. (ed.) Advances in Cryptology - CRYPTO’ 92, pp. 139-147. Springer, Berlin (1993)

Dyhrberg, A. H.: Bitcoin, gold and the dollar - a garch volatility analysis. Finance Res. Lett. 16(Supplement C): $85-92(2016)$

Eom, C., Kaizoji, T., Kang, S.H., Pichl, L.: Bitcoin and investor sentiment: statistical characteristics and predictability. Phys. A 514, 511-521 (2019)

Figa-Talamanca, G., Patacca, M.: Does market attention affect bitcoin returns and volatility? Decis. Econ. Finance 42(1), 135-155 (2019)

Figà-Talamanca, G., Patacca, M.: Disentangling the relationship between bitcoin and market attention measures. J. Ind. Bus. Econ. 47(1), 71-91 (2020)

Figà-Talamanca, G., Focardi, S., Patacca, M.: Regime switches and commonalities of the cryptocurrencies asset class. N. Am. J. Econ. Finance 57, 101425 (2021)

Figà-Talamanca, G., Focardi, S., Patacca, M.: Common dynamic factors for cryptocurrencies and multiple pair-trading statistical arbitrages. Decis. Econ. Finance, pp. 1-20 (2021)

Fry, J., Cheah, E.T.: Negative bubbles and shocks in cryptocurrency markets. Int. Rev. Financ. Anal. 47, 343-352 (2016)

Gandal, N., Hamrick, J., Moore, T., Vasek, M.: The rise and fall of cryptocurrency coins and tokens. Decis. Econ. Finance, pp. 1-34 (2021)

Garcia, D., Tessone, C.J., Mavrodiev, P., Perony, N.: The digital traces of bubbles: feedback cycles between socio-economic signals in the bitcoin economy. J. R. Soc. Interface 11(99), 20140623 (2014)

Ghorbel, A., Jeribi, A.: Investigating the relationship between volatilities of cryptocurrencies and other financial assets. Decis. Econ. Finance, pp. 1-27 (2021)

Grunspan, C., Pérez-Marco, R.: Double spend races. Int. J. Theor. Appl. Finance 21(08), 1850053 (2018)

Grunspan, C., Pérez-Marco, R.: On profitability of Nakamoto double spend. Probability in the Engineering and Informational Sciences pp. 1-15 (2021)

Hafner, C.M.: Testing for bubbles in cryptocurrencies with time-varying volatility. J. Financ. Economet. 18(2), 233-249 (2020)

Kristoufek, L.: What are the main drivers of the bitcoin price? Evidence from wavelet coherence analysis. PLoS ONE 10(4), e0123923 (2015)

Kumar, A.S., Anandarao, S.: Volatility spillover in crypto-currency markets: some evidences from GARCH and wavelet analysis. Phys. A 524, 448-458 (2019)

Kyriazis, N. A.: the Investigating diversifying or hedging nexus of cannabis cryptocurrencies with major digital currencies. Decis. Econ. Finance, pp. 1-21 (2021)

Lintilhac, P.S., Tourin, A.: Model-based pairs trading in the bitcoin markets. Quant. Finance 17(5), 703-716 (2017)

Majdoub, J., Sassi, S.B., Bejaoui, A.: Can fiat currencies really hedge bitcoin? evidence from dynamic short-term perspective. Decisions in Economics and Finance, pp. 1-28 (2021)

Mensi, W., Rehman, M.U., Al-Yahyaee, K.H., Al-Jarrah, I.M.W., Kang, S.H.: Time frequency analysis of the commonalities between bitcoin and major cryptocurrencies: portfolio risk management implications. N. Am. J. Econ. Finance 48, 283-294 (2019)

Nakamoto, S.: Bitcoin: A peer-to-peer electronic cash system. Decent. Bus. Rev., p. 21260 (2008)

Nakano, M., Takahashi, A., Takahashi, S.: Bitcoin technical trading with artificial neural network. Phys. A 510, 587-609 (2018)

Nian, L.P., Chuen, D.L.K.: Introduction to bitcoin. In: Chuen, D.L.K. (ed.) Handbook of Digital Currency, Elsevier, pp 5-30 (2015)

Platanakis, E., Sutcliffe, C., Urquhart, A.: Optimal vs naïve diversification in cryptocurrencies. Econ. Lett. 171, 93-96 (2018)

Provenzano, D., Baggio, R.: Complexity traits and synchrony of cryptocurrencies price dynamics. Decis. Econ. Finance, pp. 1-15 (2021) 
Scaillet, O., Treccani, A., Trevisan, C.: High-frequency jump analysis of the bitcoin market. J. Financ. Economet. 18(2), 209-232 (2020)

Shynkevich, A.: Bitcoin arbitrage. Financ. Res. Lett. 40, 101698 (2021)

Siu, T.K., Elliott, R.J.: Bitcoin option pricing with a SETAR-GARCH model. Eur. J. Finance 27(6), 564-595 (2021)

Tiwari, A.K., Adewuyi, A.O., Albulescu, C.T., Wohar, M.E.: Empirical evidence of extreme dependence and contagion risk between main cryptocurrencies. N. Am. J. Econ. Finance 51, 101083 (2020)

Yaya, O. S., Ogbonna, A. E., Olubusoye, O. E.: How persistent and dynamic inter-dependent are pricing of bitcoin to other cryptocurrencies before and after 2017/18 crash? Physica A: Statistical Mechanics and its Applications p 121732 (2019)

Yermack, D.: Is bitcoin a real currency? An economic appraisal. In: Chuen, D.L.K. (ed.) Handbook of Digital Currency, Elsevier, pp. 31-43 (2015)

Publisher's Note Springer Nature remains neutral with regard to jurisdictional claims in published maps and institutional affiliations. 